\title{
A Survey of Corner Detection Methods
}

\author{
Junqing Wang, ${ }^{1, *}$ and Weichuan Zhang ${ }^{2}$ \\ ${ }^{1}$ Chengdu Textile College, School of Electrical and Information Engineering, Chengdu, 611731, China \\ ${ }^{2}$ Xi'an Polytechnic University, School of Electronics and Information, Xi'an, 710072, China \\ ${ }^{*}$ Corresponding author
}

\begin{abstract}
In this paper, we present an overview of various corner detection approaches that have been proposed in the last four decades. Corner detection algorithms can be divided into intensity-based, contour-based and model-based methods. Intensity-based methods are mainly based on measuring local intensity variation of image. Contour-based methods find corners by analyzing the shape of edge contour. Model-based methods extract corners by fitting the local image into a predefined model. Meanwhile, the problems of the existing corner detection methods are given.
\end{abstract}

Keywords-corner detection; feature extraction; algorithm research

\section{INTRODUCTION}

Corners in images represent critical information in describing object features, which play a crucial and irreplaceable role in computer vision and image processing. Many computer vision tasks rely on the successful detection of corners, including 3-D reconstruction, stereo matching, object recognition and object tracking [1-4], etc.

However, it still has not a strict mathematical definition for corner; corners are usually defined as the points with low selfsimilarity or location where variations of the intensity in all directions are high. Alternatively, corners may be defined as image points where the local maxima of curvature on the edge contour or the intersection of the two of more edge curves. In the past decades, a substantial number of promising corner detection methods based upon the different corner definitions have been proposed by vision researchers. However, a complete comparative study to evaluate each of them has yet to be reported. This paper intends to fill the gap. Furthermore, multiple evaluation criteria are used to judge the performance of the detectors objectively, which include the ground truth verification [5] and the repeatability under various transformations [6].

This paper is organized as follows. Section 2 presents an overview of corner detection algorithms. The conclusions are made in section 3 .

\section{LITERATURE SURVEY OF CORNER DETECTOR}

This section presents a review of the existing literature on corner detection methods, and then a survey of previous performance evaluation criteria for corner detection is summarized.
In the literature, the terms "point feature", “dominant point”, "critical point" and "corner" are taken as equivalent. However, the terms "interest point" and "salient point" include not only "corner", but also junctions and blobs, as well as significant texture variation [7]. The existing corner detection methods can be classified into three main classes: intensity-based, contourbased and model-based methods. The presence of three categories methods have their strengths, weaknesses and expose the defects with different degree in practical application, which makes the corner detection become research hotspot in the field of computer vision and image processing.

\section{A. Intensity-Based Methods}

Intensity-based methods detect corners directly on images that mainly considered corners as points which with low selfsimilarity in an image. Moravec [8,9] found that the difference between the adjoining pixels of an edge or a uniform region of the image is small. However, the difference is significantly high in all directions at the corner. Harris and Stephens [10] developed the Moravec's idea and proposed Harris algorithm by using the first order derivative to approximate the second derivatives. For the input image I, the Harris technique first computes the derivatives of the $\mathrm{x}-, \mathrm{y}$-axes. Then, it performs convolutions of $I_{x}^{2}, I_{y}^{2}, I_{x} I_{y}$ with a Gaussian window $G_{x, y}$ to obtain an autocorrelation matrix $M$. The equations are denoted as follows:

$$
\begin{aligned}
& I_{x}=\frac{\partial I}{\partial x}, I_{y}=\frac{\partial I}{\partial y} \\
& \overline{I_{x}^{2}}=I_{x}^{2} \otimes G, \overline{I_{y}^{2}}=I_{y}^{2} \otimes G, \overline{I_{x} I_{y}}=I_{x} I_{y} \otimes G \\
& G_{x, y}=\frac{1}{2 \pi \sigma^{2}} \exp \left(-\left(x^{2}+y^{2}\right) / 2 \sigma^{2}\right) \\
& M=\left[\begin{array}{cc}
\overline{I_{x}^{2}} & \overline{I_{x} I_{y}} \\
\overline{I_{x} I_{y}} & \overline{I_{y}^{2}}
\end{array}\right]
\end{aligned}
$$

where $\otimes$ is a convolution operator, $\sigma$ is Gaussian scale. The corner response $R$ is calculated by the matrix $M$

$$
R=\alpha \beta-k(\alpha+\beta)^{2}
$$

where $\alpha, \beta$ are eigenvalues of $M(\alpha \geq \beta), k$ is false corner response suppression.

Corners are local anisotropic features of image. The key of the intensity-based corner detection is to extract local grayvariation and structural information effectively. It was 
indicated [5] that the two partial derivatives cannot describe the local gray variation completely, which results in the Harris detector only extract L-type corners well [11]. Meanwhile, the selection of corner response suppression affects the accuracy of corner detection. To amend the aforementioned problems, the adaptive false corner response suppression parameter function [12], adaptive smoothing-scale change [13], combinations of first- and second- order derivatives [14], local weight coefficient [15], applying log-log scale to gradient vectors [16], local gradient covariance matrix [17], improved non-maximum suppression [18] and adaptive structure tensor [19] are used to improve the corner detection performance at some extent, respectively. With the development of multi-scale geometric analysis technology, the local gray variation and geometric structure of image can be described more precisely. Multi-scale and multi-resolution[20,21] technology is widely used in corner detection algorithm to reduce the effect of noise and improve the accuracy of image feature information extraction and corner location.

It is well-known that the natural image features may appear in different ways depending upon the scale of observation. This insight leads to the multi-scale representation [22-24] of image data, which became one of the main means to describe the local gray-variation and geometric structures of image and extract and localize corners. One of the first systems to extract corners in scale-space was proposed in [25], which built a Laplacian pyramid for coarse-to-fine feature extraction. Since then, many methods for selecting corners in scale-space have been proposed. Wavelets [26, 27], Gabor wavelet [28, 29] and LogGabor wavelet [30] are applied to detect corners; multi scales and orientations are used to depict the local gray variation, and then the geometry information are embedded into the framework of Harris algorithm.

However, multi-scale algorithms usually produce a substantial expansion of the amount of data to be processed. This problem can be addressed by automatically selecting the appropriate scales for feature extraction, which is also referred to the scale invariance. Lindeberg [31] presented a systematic methodology of automatic scale selection. The idea is to select the characteristic scale of a local structure, for which a given function attains an extrema over scales. In the same year, the Gabor wavelet was used to extract multi-scale and multidirection gray-level change information [32, 33], which improved the detection performance. Lowe [34] proposed a scale invariant feature transform (SIFT), which combines a scale invariant region detector and a descriptor based upon on the gradient distribution in the detected region. Scale invariant corners are extracted by convolving the image with differenceof-Gaussian (DoG) filters at a variety of scales and selecting local maxima in scale space. Mikolajczyk and Schmid [35, 36] applied a multi-scale version of the Harris detector to localize corners in space and then employ Lindeberg's scheme [31] for scale selection and affine adaption. Furthermore, Mikolajczyk and Schmid [37] extended the SIFT descriptor [34] and presented gradient location and orientation histogram (GLOH) detector. Inspired by SIFT [34] detector, Bay et al. [38] presented SURF detector that locates the feature points at which the determinant of the Hessian reaches its maximum. Meanwhile, the low complexity is enabled by employing the box filters and the integral images. Marimon et al. [39] proposed DARTs detector; the weighted triangle responses are used to approximate the second derivative of Gaussian kernel, which corresponds to the elements of Hessian matrix. Nevertheless, the aforementioned scale invariance detectors [31-39] are based upon the linear partial differentiation on Gaussian scale space. It is considered that Gaussian smoothing leads to image blurring. Recently, scale invariance detectors $[40,41]$ based upon non-linear partial differential equation (PDE) have been proposed. Alcantarilla et al. [41] proposed an accelerated version of KAZE to reduce the computation complexity. Nowadays, an optimized architecture [42] based upon the field programmable gate arrays (FPGAs) is proposed, which makes the Harris detector can real-time corner detection.

\section{B. Contour-Based Methods}

Contour-based methods first obtain image's planar curves by some edge detector (e.g., Canny edge detector [43]) and then analyze the properties of the contours' shape to detect corners. These methods first find image's edge map by some edge detector, and then extract closed or open edge contours. Thereafter, the points of local curvature maxima, line intersects or rapid changes in the edge direction are marked as corners.

Attneave [44] found the curvature is relatively large at the corners by observing the shape of edge contour. Early methods first operate image segmentation, and then extract edge Freeman chaining. Rosenfeld and Johnston [45] presented a corner detector using $\mathrm{K}$-cosine as the estimation of the curvature value for a point on a curve. An improved version [46] of [45] was proposed by using K-cosine average. Alternately, the corners are labeled as the isolated discontinuities points in the mean slope [47, 48], which are computed in the Freeman chain. A similar technique was proposed by Beus and Tiu [49], where both local curvature averaging and determination of isolated points are taken into account. Asada and Brady [50] used the Gaussian smoothing and cubic B-splines to calculate the K-curvature and label the corner points. Cooper [51] introduced an operator that the pixel coordinates of chain-code are used to estimate curvature and detect corners. Arrebola et al. introduced three different corner detectors based on local [52], circular [53] histogram of contour chain code, and multi-resolution [54] chain code linking. Instead of direct detecting on the curve, cubic polynomials are fit to curves and discontinuities are detected in curves to localize corners [55-57]. Alternatively, Kitchen and Rosenfeld [58] developed a corner measure based upon the change of gradient direction along an edge contour multiplied by the local gradient magnitude as follows:

$$
C_{K R}(x, y)=\frac{I_{x x} I_{y}^{2}-2 I_{x y} I_{x} I_{y}+I_{y y} I_{x}^{2}}{I_{x}^{2}+I_{y}^{2}} .
$$

Wang and Brady [59] developed an algorithm by computing image curvature that is proportionate to the second derivative along the edge tangential and is inversely proportional to the edge strength.

Asada and Brady [60] extended the scale space concept to represent significant changes in curvature along a planar curve 
and then detect corners. Mokhtarian and Mackworh [61, 62] developed the curvature scale space (CSS) technique to detect corners by finding curvature zero-crossing points of curves. Rattarangsi and Chin [63] extended the CSS technique to detect and localize the corners on edge contours. The 'L', 'END' and 'STAIR' corner models are constructed; and then the scale space map of corners are investigated by Gaussian convolution multi scale representation of the models. However, the tree construction and parsing based upon the complete scale-space resulted in high computational complexity. In view of the CSS technology can effectively recover the geometric features of planar curve in multi-scale space [62], Mokhtarian and Suomela [64] proposed a CSS corner detector. For a given parametric vector equation of a planar curve $\Gamma(u)=\{x(u), y(u)\}$, the curvature is defined as

$$
K(u, \sigma)=\frac{\dot{X}(u, \sigma) \ddot{Y}(u, \sigma)-\ddot{X}(u, \sigma) \dot{Y}(u, \sigma)}{\left[\dot{X}(u, \sigma)^{2}+\dot{Y}(u, \sigma)^{2}\right]^{3 / 2}}
$$

where

$$
\begin{array}{ll}
\dot{X}(u, \sigma)=x(u) \otimes \dot{g}(u, \sigma) & \ddot{X}(u, \sigma)=x(u) \otimes \ddot{g}(u, \sigma) \\
\dot{Y}(u, \sigma)=y(u) \otimes \dot{g}(u, \sigma) & \ddot{Y}(u, \sigma)=y(u) \otimes \ddot{g}(u, \sigma)
\end{array}
$$

and $\otimes$ is the convolution operator, $\sigma$ is the scale factor, $\dot{g}(u, \sigma)$ and $\ddot{g}(u, \sigma)$ are the first- and second derivatives of Gaussian $g(u, \sigma)$, respectively. The corners are localized at the maxima of absolute curvature of planar curve, and then corners are tracked from the highest scale to the lowest scale to improve the localization error. To improve corner localization and noise suppression, an enhanced CSS algorithm [65] is proposed by using different scales of the CSS for contours with different length.

It is indicated [66-76] that the existing CSS corner detectors [60-65] suffer from three main problem. First, The curvature estimator is sensitive to the local variation or noise on contours. Second, Gaussian scale selection is in a dilemma. Third, the determintation of threshold. Ray and Pandyanp [66] presented an adaptive corner detection algorithm. Local variance of curvature is computed to measure the variation in the roughness of a curve, and then the technique for smoothing a curve adaptively is used to solve the problem of Gaussian scale selection. Zhong and Liao [67] proposed direct curvature scale space (DCSS) technique based on the earlier work form [63] to improve the computational complexity. Zhang et al. [68] introduced multi scale curvature product technique to enhance the noise robustness. Local extremes of the curvature product at three different smoothing scales are marked as corners, which makes strong corners more distinguishable from the weak corners. He and Yung [69] used an adaptive curvature threshold in a dynamic region of support to judge corners. Awrangjeb and Lu [70] proposed an improved CSS corner detector which is invariant to geometric transformations. The affine-length instead of arc-length parameterization is used to compute the curvature. Alternatively, chord-to-distance accumulation technique [71] is applied to compute curvature and detect corners [72]. Zhang et al. [73] introduced an operator for detection based on evolution difference of Gaussian scale space, which can well reflect the change of the corners between the evolved curves. Pedrosa and Barcelos [74] proposed an adapted smoothing scale corner detection algorithm based on anisotropic diffusion. Zhang et al. [75] proposed a determinant of the gradient correlation matrix operator, a $1 \times 1$ neighborhood around each edge point is used to estimate curvature and extract corners. Rimon and Robert [76] presented the corner detection by changing the gradient direction of edge pixel. Zhang et at. [77] extended the Moravec's idea; the imaginary part of Gabor filters are used to smooth each edge pixel and its surrounding pixels, the points which have large gray variation at each orientation are marked as corners.

It is noticed that the performance of edge-based corner detectors depend upon the edge detection largely. Furthermore, edge contours are often imprecise in the neighborhood of a junction, so the edge-based corner detectors are difficult to characterize the junction accurately.

\section{Model-Based Methods}

Model-based methods extract corners by fitting a small patch of an image into a predefined model. Different approaches are used to compare the deviation between the target and template. The target point would be regarded as a corner point if it matches one of the templates.

Guiducci [78] presented an algorithm for the description of the corner points by a parameters model of three basic physical characteristics of corner points namely the aperture, amplitude and blur. Rargarajan et al [79] created the response of L-shape corner and approximated all possible corners with 13 types of mark. Singh and Shneier [79] proposed that using different corner type combinations to image matching filtering. Rohr [81] extended the wedge corner model to $\mathrm{T}$ type, $\mathrm{Y}$ type and $\mathrm{X}$ type and the corner is determined to be the intersection of multiple homogeneous regions. Deriche and Giraudon [81] utilized the Gaussian kernel characterize wedge and Y type corner model and analyze the characterization characteristics, and Beaudet's [81]method is used to correct the corner location. Due to the use of 2D Gaussian smoothing function, the algorithm is timeconsuming, which is improved by replacing the Gaussian function to exponential function in the later work of Blaszk and Deriche [84]. Smith and Brady [85] proposed a non-linear filtering method SUSAN to be used in corner detection and noise reduction. The basic idea is to find the number of pixels that have similar intensity with centre point in the neighbourhood. If the number below a threshold (the author suggested 25 in a mask of 37 pixels), then after false positives test and non-maximum suppression, the corner can be detected. Trajkovic and Hedley [86] reduced the computation complexity by only detect the variation of image intensity along the lines passing through the centre point within a neighbourhood of the point, but the result is unstable and has the problem of false corners detection. Baker et al[87] presented an algorithm to construct detectors automatically for arbitrary parametric features including edges, lines, corners and junctions. The author convert and store each feature into a parametric manifold in a low dimensional subspace of a Hilbert space and then is compared within a window respectively during 
detection. If the vector of intensity value in a window is similar enough to a feature manifold, feature in the window can be detected. In the meanwhile, parameters of feature model can be recovered using the closest point on the feature manifold. Shui and Zhang [88] constructed corner models and utilized the anisotropic Gaussian directional derivatives[89]to detect and classify corners. Florentz and Aldea[90] predicted the optimal threshold for corner detection by using a predefined angular point model that significantly reduced detection time and improved robustness of scene changes.

In general, three different categories corner detection methods follow their own development paths, and there are relatively few techniques to integrate each other. In recent years some methods based on machine learning and fusion are introduced into corner detection algorithm and the detection performance is improved.

\section{CONCLUSION}

This paper presents a review of the existing corner detection algorithms. Their corresponding advantages and disadvantages are discussed in detail. The aim of this paper is to raises readers' interest in corner detection research.

\section{ACKNOWLEDGMENTS}

This work was supported by the National Natural Science Foundation of China (No. 61401347) and by the Natural Science Basic Research Plan in Shaanxi Province of China (No. 2016JM6013).

\section{REFERENCES}

[1] Ambar Dutta, A. Kar, and B.N. Chatleri, "A new approach to corner matching from image sequence using fuzzy similarity index,” Pattern Recognition Letters. 32(5):712-720, 2017

[2] S. Gauglitz, T. Hollerer, M. Turk, "Evaluation of interest point detectors and feature descriptors for visual tracking," International Journal of Computer Vision, 94:335-360, 2011.

[3] Krystian Mikolajczyk and Cordelia Schmid, "Scale \&affine invariant interest point detectors,” International Journal of Computer Vision, 60(1): 63-86, 2004

[4] P. Mainli, G. Lafruit, Q. Yang, B. Geelen, L. V. Gool, R. Lauwereins, "SIFER: Scale-invariant feature detector with error resilience," International Journal of Computer Vision, 104: 172-197, 2013.

[5] Peng-Lang Shui, Wei-Chuan Zhang, "Corner detection and classification using anisotropic directional derivative representations,” IEEE Trans. on Image Processing, 22(8): 3204-3219, 2013.

[6] M. Awrangjeb and G. Lu, "Robust image corner detection based on the chord-to-point distance accumulation technique," IEEE Trans. on Multimedia, 10(6): 1059 -1072, 2008.

[7] K. Mikolajczyk and C. Schmid, "Indexing based on scale invariant interest points,” Proc. Eighth Int. Conf. Computer vision, pp. 525-531, 2015.

[8] H. P. Moravec, “Towards automatic visual obstacle avoidance,” Proc. 5th International Joint Conf. on Artificial intelligence, pp. 584, 1977.

[9] H. P. Moravec, "Visual mapping by a robot rover," International Joint Conf. on Artificial intelligence, pp.598-600, 1979.

[10] C. Harris and M. Stephens, "A combined corner and edge detector," Proc. Alvey Vision Conf., Univ.Manchester, pp. 147-151, 1988.

[11] J. A. Noble, “finding corners,” Image Vis. Comput., vol. 6, pp.121-128, 1988.

[12] Zhiqiang Zheng, Han Wang, Eam Khwang Teoh, “Analysis of gray level corner detection,” Pattern Recognition Letters, 20(6): 149-162, 1999.
[13] Dufournaud, Y., Schmid, C., and Horaud, R. Matching images with different resolutions. In Proceedings of the Conference on Computer Vision and Pattern Recognition, Hilton Head Island, South Carolina, USA, pp. 612-618, 2000.

[14] Wang, H., Brady, M., "Real-time corner detection algorithm for motion estimation,” Image and Vision Computing, 13 (9), 1995.

[15] J.-B. Ryu, C.-G. Lee, H.-H. Park, "Formula for Harris corner detector," Electronic letters, 47(3):5-6, 2011.

[16] J.-B. Ryu, H. -H. Park, "Log-log scale Harris corner detector," Electronic letters, 46(24):21-22, 2010.

[17] S. Ando, "Image field categorization and edge/corner detection from gradient covariance," IEEE Trans. Pattern Anal. Mach. Intell., 22(2):179-190, 2000.

[18] P. Mainali, Q. Yang, G. Lafruit, L. V. Gool, R. Lauwereins, "Robust low complexity corner detector," IEEE Trans. Circuits and Systems for Video Technology, 21(4): 435-446, 2011.

[19] Bongjoe Kim, Jihoon Choi, Yongwoon Park, Kwanghoon Sohn, "Robust corner detection based on image structure," Circuits, system and signal processing, 31:1443-1457, 2012.

[20] J.L. Crowley, A.C. Sanderson. Multiple resolution representation and probabilistic matching of 2D gray-scale shape[J]. IEEE Trans. PAMI,1987, 9(1):113-121.

[21] Lindeberg, T. Scale-space theory in computer vision[M].Kluwer Academic Publishers,1994.

[22] Lindeberg, T. "Scale-space for discrete signals,” IEEE Trans. Pattern Anal. Mach. Intell., 12(1):234-254, 1990.

[23] Lindeberg, T. "Scale-space theory in computer vision," Kluwer Academic Publishers, 1994.

[24] J. L. Crowley and A. C. Sanderson, "Multiple resolution representation and probabilistic matching of 2D gray-scale shape,” IEEE Trans. Pattern Anal. Mach. Intell., 9(1):113-121, 1987.

[25] P. J. Burt, E. H. Adelson, “The Laplacian pyramid as a compact image code,” IEEE Trans.on Communications, 9(4):532-540, 1983.

[26] C. H. Chen, J. S. Lee, and Y. N. Sun, "Wavelet transformation for graylevel corner detection,” Pattern Recognition, 28(6): 853-861, 1995.

[27] Nicu Sebe, Michael S. Lew, “Comparing salient point detectors,” Pattern Recognition letters, 24: 89-96, 2003.

[28] A. Quddus and M. M. Fahmy, “Corner detection using Gabor-type filtering,” in Proc. IEEE ISCAS, 1998, vol.4, pp.150-153.

[29] J. F. Valdivia, J. Garcia, J. Martinez-Baena, and X. R. Fdez-Vidal, "The selection of natural scales in 2-D images using adaptive Gabor filtering," IEEE Trans. Pattern Anal. Mach. Intell., 20(5): 458-469, 1998.

[30] X-T Gao, F. Sattar, and R. Ventateswarlu, "Multiscale corner detection of gray level images based on log-Gabor wavelet transform,” IEEE Trans. Circuits and Systems for Video Technology, 17(7): 868-875, 2007.

[31] Lindeberg, T. "Feature detection with automatic scale selection," International Journal of Computer Vision, 2(30):79-116, 1998.

[32] A.Quddus, M.M. Fahmy. Corner detection using Gabor-type filtering[A].Proceedings of the IEEE international Symposium Circuits and Systems[C] Monterey Conference Center Monterey :1998.150-153.

[33] J.F. Valdivia, J. Garcia. The selection of natural scales in 2-D images using adaptive Gabor filtering[J].IEEE Trans. PAMI, 1998,20(5): 458469.

[34] D. Lowe, "Distinctive image features from scale-invariant keypoints," International Journal of Computer Vision, 2(60):91-110, 2004.

[35] K. Mikolajczyk and C. Schmid, "Indexing based on scale invariant interest points,” Proc.Eighth Int. Conf. Computer vision, pp. 525-531, 2001.

[36] K. Mikolajczyk and C. Schmid, “An affine invariant interest point detector,” Proc. Int.Seventh Conf. Computer vision, pp. 525-531, 2002.

[37] K. Mikolajczyk, C. Schmid, “A performance evaluation of local descriptors,” IEEE Trans. Pattern Anal. Mach. Intell., 27(10):1615-1630, 2005.

[38] H.Bay,A.Ess,T.Tuytelaars,L.VanGool, "Speeded-up robust features (SURF)," Computer Vision and Image Understanding, 110(3):346-359, 2008. 
[39] D. Marimon, A. Bonnin, T. Adamek, R. Gimeno, "Darts: Efficient scale space extraction of daisy keypoints,” Proc. Intl Conf. on Comp. Vis. and Pat. Recog (2010) 2416-2423.

[40] P. F. Alcantarilla, A. Bartoli, A. J. Davison, "Kaze features,” Proc. Eur. Conf. on Pat. Recog,(ECCV) 214-227, 2012.

[41] P. F. Alcantarilla, J. Nuevo, A. Bartoli, Fast explicit diffusion for accelerated features in nonlinear scale spaces, Proc. of the Brit.Mach. Vis. Conf. (BMVC), 2012.

[42] P. R. Possa, S. A. Mahmoudi, Naim Harb, C. Valderrama, P. Manneback, "A multi-resolution FPGA-based architecture for real-time edge and corner detection,” IEEE Trans. on Computers, 63(10):2376-2388, 2014.

[43] Canny J, “A computational approach to edge detection,” IEEE Trans. Pattern Anal. Mach. Intell., 8(6):679-698, 1986.

[44] F. Attneave, "Some informational aspects of visual perception," Psychological Review, vol. 61, pp. 183-193, 1954.

[45] A. Rosenfeld and E. Johnston, “Angle detection on digital curves,” IEEE Transactions on Computers, vol. C-22, pp. 875-878, 1973.

[46] A. Rosenfeld and J. S. Weszka, “An improved method of angle detection on digital curves,” IEEE Transactions on Computers, 24(9):940-941, 1975.

[47] H. Freeman, "Computer processing of line drawing images," Surveys, 6(1):57-97, 1974.

[48] H. Freeman and L. S. Davis, "A corner-finding algorithm for chaincoded curves,” IEEE Transactions on Computers, vol. 26, pp. 297-303, 1977.

[49] H. L. Beus and S.S.H. Tiu, "An Improved Corner Detection Algorithm Based on Chain-Coded Plane Curves,” Pattern Recognition, 20(3):291296, 1987.

[50] Asada, H., Brady, M., “The curvature primal sketch,” IEEE Trans. Pattern Anal. Machine Intell. 8(1): 2-14, 1986.

[51] Cooper J, Venkatesh S, Kitchen L., "Early Jump-out Corner Detectors," IEEE Trans. Pattern Anal. Mach. Intell., 15(8): 823-828, 1993.

[52] F. Arrebola, A. Bandera, P. Camacho, F. Sandoval, "Corner detection by local histograms of contour chain code," Electronic Letters, 32(21):1796-1771, 1997.

[53] F. Arrebola, P. Camacho, A. Bandera, F. Sandoval, "Corner detection and curve representation by circular histograms of of contour chain code,” Electronic Letters, 35(13):1065-1067, 1999.

[54] F. Arrebola, F. Sandoval, "Corner detection and curve segmentation by multi-resolution chain-code linking,” Pattern Recognition, 38(7):15961614, 2005.

[55] D. J. Langridge, "Curve encoding and detection of discontinuities," Computer Graphics Image Processing, vol. 20, pp. 58-71, 1982.

[56] G. Medioni and Y. Yasumoto, "Corner Detection and Curve Representation Using Cubic B-Splines,” Computer Vision, Graphics, and Image Processing, 39(3):279-290, 1987.

[57] Hsin-Teng, Hu W C. “A Rotationally Invariant Two-phase Scheme for Corner Detection,” Pattern Recognition, 28(3):819-828, 1996.

[58] L. Kitchen and A. Rosenfeld, “Gray-Level Corner Detection,” Pattern Recognition Letters, 1(2): 95-102, 1982.

[59] H. Wang, M. Brady, "Real-time corner detection algorithm for motion estimation [J],” Image and Vision Computing, 13(9):695-704, 1995.

[60] Asada, H. and Brady, M. “The curvature primal sketch,” IEEE Trans. Pattern Anal. Mach. Intell., 8(1):2-14, 1986.

[61] Mokhtarian F, Mackworth A K. "Scale-based description and recognition of planar curves and two-dimensional shapes,” IEEE Trans. Pattern Anal. Mach. Intell., 8(1):34-43, 1986.

[62] Mokhtarian F, Mackworth A K. "A theory of multi-scale curvaturebased shape representation for plane curves,” IEEE Trans. Pattern Anal. Mach. Intell., 14(8):789-805, 1992.

[63] Anothai Rattarangsi, Roland T. Chin, "Scale-based detection of corners of planar curves,” IEEE Trans. Pattern Anal. Mach. Intell., 14(4):430450, 1992.

[64] Mokhtarian F , Suomela R., "Robust image corner detection through curvature scale space," IEEE Trans. Pattern Anal. Mach. Intell., 20(12):1376-1381, 1998
[65] Mokhtarian F , Mohanna F., "Enhancing the curvature scale space corner detector,” Proc. Scandinavian Conf. on Image Analysis, pp.145152, 2001.

[66] B. K. Ray and R. Pandyan, “ACORD-an adaptive corner detector for planar curves,” Pattern Recognistion, 36(3):703-708, 2003.

[67] Baojiamg Zhong, Wenhe Liao, "Direct curvature scale space: theory and corner detection,” IEEE Trans. Pattern Anal. Mach. Intell., 29(3):508512, 2007.

[68] Xiaohong Zhang, Ming Lei , Dan Yang, Yuzhu Wang, Litao Ma, "Multi-scale curvature product for robust image corner detection in curvature scale space,” Pattern Recognition Letters, 28(7):545-554, 2007.

[69] Xiaochen He, N. H. C. Yung, "Corner detector based on global and local curvature properties,” Optical Engineering, 47(5):1-12, 2008.

[70] M. Awrangjeb and G. Lu, “An improved curvature scale-space corner detector and a robust corner matching approach for transformed image identification,” IEEE Trans. Image Processing, 17(2): 2425-2441, 2008.

[71] J. H. Han and T. T. Poston, "Chord-to-point distance accumulation and planar curvature: A new approach to discrete curvature," Pattern Recognition Letter, 22(7):1133-1144, 2001.

[72] Mohammad Awrangjeb, Guojun Lu, "Robust image corner detection based on the chord-to-point distance accumulation technique," IEEE Trans. on Multimedia, 10(6): 1059-1072, 2008.

[73] X. Zhang, H.Wang, M. Hong, L. Xu, D. Yang, and B. C. Lovell, "Robust image corner detection based on scale evolution difference of planar curves,” Pattern Recognition Letters, 30(1):449-455, 2009

[74] G. V. Pedrosa and C. A. Z. Barcelos, "Anisotropic diffusion for effective shape corner point detection,” Pattern Recognition Letters, 31(12):1658$1664,2010$.

[75] X. Zhang, H. Wang, A. W. B. Smith, X. Ling, B. C. Lovell, and D. Yang, "Corner detection based on gradient correlation matrices of planar curves,” Pattern Recognition, 43(4):1207-1223, 2010.

[76] Rimon Elias, Robert Laganière. JUDOCA: Junction detection operator based on circumferential anchors[J]. IEEE Trans. Image Processing, 2012, 21(4):2109-2118

[77] Wei-Chuan Zhang, Fu-Ping Wang, Lei Zhu, Zuo-Feng Zhou, “Corner detection using Gabor filters,” IET Image Processing, 8(11):639-646, 2014.

[78] Guiducci, Antonio. "Corner characterization by differential geometry techniques." Pattern Recognition Letters, 8(5):311-318, 1988.

[79] Rargarajan K, Shah M, Brackle D V. "Optimal corner detection," Computer Vision Graphics linage Process, 1989, 48: 230-245.

[80] A. Singh, M. Shneier. Grey level corner detection: a generalization and a robust real time implementation[J].Computer Vision Graphics Image Processing,1990,51(1): 54-69.

[81] K. Rohr, "Recognizing corners by fitting parametric models," International Journal of Computer Vision, 9(3):213-230, 1992.

[82] R. Deriche, G. Giraudon. A computational approach for corner and vertex detection[J].International Journal of Computer Vision,1993,10(2):101-124.

[83] Beaudet, Rotational invariant image operators[A].Intern. Conf. Pattern Recognition[C].Tokyo:1978.579-583.

[84] T. Blaszka, R. Deriche. Recovering and characterizing image features using an efficient model based approach[A].Conference of Computer Vision and Pattern Recognition[C], Proceedings CVPR'93: 1994: 530535

[85] Smith S M, Brady J M. "SUSAN-A new approach to low level image processing,” International journal of computer vision, 1997, 23(1): 45-78

[86] M. Trajkovic, M. Hedley. "Fast corner detection," Image and vision computing, 1998, 16(2): 75-87.

[87] Baker S, Nayar S K, Murase H, "Parametric feature detection," International Journal of Computer Vision, 1998, 27(1): 27-50.

[88] E. Rosten, R. Porter. Faster and better: A machine learning approach to corner detection [J].IEEE Trans. PAMI,2010, 32(1):105-117.

[89] SHUI Peng-lang, ZHANG Wei-chuan. Corner detection and classification using anisotropic directional derivative representations[J]. IEEE Trans. on Image Processing, 2013, 22(8): 3204-3219. 
[90] GASPARD F, EMANUEL A. Super FAST: Model-based adaptive corner detection for scalable robotic vision[C].IEEE Conference Publications, 2014, 1003-1010 